Intravenöse Eisensubstitution bei Hämodialysepatienten

\section{IDIRA-Studie: \\ Niedermolekulares Eisendextran effizienter als Eisenglukonat}

Die jüngst veröffentlichten Daten der IDIRAStudie (Iron Dextran in Renal Anaemia) [1] zeigen, dass sich niedermolekulares Eisen-IIIDextran zur parenteralen Eisensubstitution nicht nur durch seine hohe Wirksamkeit, sondern auch durch gute Verträglichkeit und hohe Sicherheit auszeichnet: Bei 221 stabilen Hämodialysepatienten, die von Eisenglukonat $\left(\right.$ Ferrlecit $\left.{ }^{\circledR}\right)$ auf niedermolekulares Eisen-IIIDextran $\left(\mathrm{CosmoFer}^{\circledR}\right)$ umgestellt wurden, konnte bei gleicher Eisendosis eine höhere Wirksamkeit hinsichtlich der Steigerung der
Ferritin- und Hämoglobinwerte festgestellt werden. Darüber hinaus verbesserte das niedermolekulare Eisendextran den inflammatorischen Status der Patienten. Bei keinem der Studienteilnehmer traten unerwünschte Arzneimittelwirkungen auf, niemand brach die einjährige Studie vorzeitig ab.

Eine Vorrausetzung, Patienten mit renaler Anämie zu behandeln, ist ein adäquater Eisenhaushalt, der häufig durch eine zusätzliche Substitution mit intravenös verabreichtem Eisen erreicht wird. Hierfür stehen Eisensaccharat und Eisenglukonat sowie niedermolekulares Eisen-III-Dextran zur Verfügung. Letzteres hat die höchste Komplexstabilität [2] aller in Deutschland zugelassener i.v. Eisenpräparate und führt zur geringfügigsten Freisetzung von Eisen außerhalb des physiologischen Speichersystems [3]. Es induziert im Vergleich zu
Eisensaccharat und Eisenglukonat nur sehr geringen oxidativen Stress $[4,5]$ Deswegen ermöglicht CosmoFer ${ }^{\circledR}$ eine Gesamtdosisinfusion von bis zu $20 \mathrm{mg}$ Eisen/kg Körpergewicht, wogegen die maximale Einzeldosis von Ferrlecit bei $62,5 \mathrm{mg}$ liegt.

Quellen

1 Rath T et al., Kongress für Nephrologie 2007, München, Poster So-1-P21

2 Langguth P. et al. Deutsche Pharmazeutische Gesellschaft Jahrestagung 2003, Poster T25

3 Geisser P. et al. Arzneimittelforschung 1992;42 1439-1452

4 Agarwal L. Sidney Int 2006;69(7):1259-1263

Weitere Informationen bei

Teva Deutschland GmbH

PhD Sandra Nestler

Waldecker Straße 11, 64546 Mörfelden-Walldorf Tel. +49 6105 97789-701

cosmofer@teva.de
Studie von CSL Behring an Tiermodellen zeigt:

Entwicklungsmöglichkeit des rekombinanten Faktors VIla mit verlängerter Halbwertzeit bei gleich bleibender biologischer Aktivität

Auf der Jahrestagung 2007 der ASH stellte CSL Behring Ergebnisse einer präklinischen Studie vor, die zum ersten Mal zeigen, dass eine genetische Fusion des Faktors VIIa (FVIIa) an menschliches Albumin möglich ist, wodurch die Halbwertszeit dieses therapeutischen Proteins unter Beibehaltung seiner biologischen Aktivität verlängert wird. Die Studienergebnisse zeigten eine um das 6- bis 9-fache verlängerte Halbwertszeit des rFVIIa-Fusions- proteins im Vergleich zum Wildtyp rFVIIa. Weiterhin wies das rFVIIa-Fusionsprotein eine mit dem Wildtyp rFVIIa vergleichbare biologische Aktivität auf.

Der rFVIIa kann zur Kontrolle von Blutungsepisoden bei Hämophiliepatienten mit Inhibitoren eingesetzt werden. Diese Patienten entwickeln eine Immunreaktion, die den substituierten Gerinnungsfaktor vom Stoppen einer Blutungsepisode abhält. Der rFVIIa hat jedoch nur eine kurze Halbwertszeit von ca. 2,5 h. Dadurch sind mehrere Injektionen erforderlich, die sowohl für Ärzte als auch für Patienten besonders bei operativen Eingriffen unangenehm sind.

Das rFVIIa-Fusionsprotein ist das Ergebnis des ersten Ausfluges von CSL Behring in die Forschungswelt der Techno- logie von rekombinanten Koagulationsprodukten.

Diese Daten weisen darauf hin, dass dieses rekombinante FVIIa-Albuminfusionsprotein einen ersten Schritt in Richtung einer patientenfreundlicheren Behandlungsoption für Hämophilie-Patienten mit Inhibitoren darstellt.

Quellen

1 Blood, Vol. 110, 11, nov. 16, 2007, \# 3142

Weitere Informationen bei

CSL Behring GmbH

Dr. Claudia Zacharias

Philipp-Reis-Straße 2, 65795 Hattersheim

Tel. +4969 305-80212

Claudia.Zacharias@cslbehring.com

\title{
Ticker+++ Ticker+++ Ticker+++ Ticker+++ Ticker+++ Ticker+++ Ticker+++
}

GlaxoSmithKline. Die neue 1,5-mg-Dosierung des selektiven Faktor-Xa-Hemmers Fondaparinux-Natrium (Arixtra ${ }^{\circledR}$ ) ist jetzt auch für Patienten mit Niereninsuffizienz verfügbar. Dadurch ergibt sich eine klare, einfache Dosierung: Bei Patienten mit einer Kreatinin-Clearance zwischen 20 und $50 \mathrm{ml} / \mathrm{min}$ ist die Dosierung auf $1,5 \mathrm{mg} 1 \times$ täglich zu reduzieren. Für alle anderen Patienten ist die Standarddosis in der Prophylaxe unverändert 2,5 $\mathrm{mg} 1 \mathrm{x}$ täglich.

Weitere Informationen bei

GlaxoSmithKline GmbH \& Co. KG

Unternehmenskommunikation

Dr. Anke Helten

Anke.Helten@gsk.com
Wyeth Pharma. Kürzlich wurden die ersten Zwischenergebnisse der Pharmakovigilanz-Untersuchung zu ReFacto ${ }^{\circledR}$ von Pollmann et al. veröffentlicht. Diese bestätigen eindrucksvoll die Wirksamkeit und Sicherheit des B-Domänen befreiten rekombinanten Faktor VIII bei zuvor unbehandelten und behandelten Patienten mit Hämophilie A.

Weitere Informationen bei Wyeth Pharma GmbH Frau Ulrike Schievelbein SchievU@ wyeth.com
Ortho Biotech. Neueste beim Internationalen Myelomworkshop auf Kos/Griechenland vorgestellte Daten zeigen: Bortezomib (Velcade ${ }^{\circledR}$ ), die stärkste Monosubstanz beim multiplen Myelom, ist unter entsprechender medizinischer Überwachung auch bei Niereninsuffizienz eine verträgliche und effektive Therapieoption.

Weitere Informationen bei

Haas \& Health Partner GmbH

Yasmin König

koenig@ haas-health.de 